\title{
Article original / Original article \\ Problématique de l'intoxication par un rodenticide non autorisé à la commercialisation en Algérie : identification de sa matière active
}

\section{The problem of poisoning by a rodenticide not authorised for commercialisation in Algeria: Identification of its active ingredient}

\author{
Fatima-Zohra Hadjadj Aoul ${ }^{1 \star}$, Radia Zamoum² ${ }^{2}$ Amel Aissani ${ }^{2}$, Farida Aliane ${ }^{1}$, Wafa Idir $^{1}$, \\ Rania Abtroun ${ }^{1}$, Mohamed Reggabi ${ }^{3}$, Bachra Alamir ${ }^{2}$ \\ ${ }^{1}$ Laboratoire de Toxicologie du Centre Hospitalo Universitaire Bab El Oued Alger, 53 boulevard Said Touati, Alger, Algérie \\ 2 Centre National de Toxicologie, route petit staoueli NIPA Dely Brahim, Alger, Algérie \\ ${ }^{3}$ Laboratoire de Biologie- Toxicologie, Etablissement Hospitalier Spécialisé Ait Idir, boulevard Abderrezak Hadad, Alger, Algérie
}

\begin{abstract}
Résumé - Objectif : 1136 cas d'intoxications volontaires ou accidentelles ont été enregistrés au Centre Antipoison du CHU Bab El Oued d'Alger durant la décennie (1998-2008), par un produit rodenticide commercialisé sous le nom de «Rat Killer» et sans mention de sa composition. La symptomatologie décrite comporte essentiellement des signes cholinergiques. Afin d'élucider cette ambiguïté, l'identification du produit s'imposait. Méthodes : Trois techniques chromatographiques ont été utilisées : chromatographie sur couche mince, chromatographie en phase gazeuse couplée à la spectrométrie de masse et chromatographie liquide couplée à la spectrométrie de masse. Résultats : L'analyse du produit confirmée par des méthodes différentes et complémentaires, met en évidence la présence de Carbofuran, insecticide de la famille des carbamates inhibiteurs de l'activité cholinéstérasique. Conclusion : l'identification du produit a permis de dénoncer une fraude et d'interpeller les pouvoirs publics quant à la commercialisation illégale et informelle de ces pesticides détournés de leur usage initial, et qui plus est interdits à l'importation en Algérie. Par ailleurs, cela a conforté le clinicien dans la prise en charge de cette intoxication.
\end{abstract}

Mots clés : Intoxication, signes cholinergiques, matière active, méthodes chromatographiques, identification

Abstract - Objectives: 1136 cases of voluntary or accidental intoxications have been registered at the anti-poison centre of Bab El Oued Academic Hospital during this last decade. These intoxications were due to a rodenticide product marketed under the name "Rat Killer" without any information about its components. The reported symptoms were essentially cholinergic signs. The aim of the study was to identify the "Rat Killer"s components. Method: Three chromatographic techniques were used: thin-layer chromatography, liquid chromatography coupled with mass spectrometry and gas chromatography coupled with mass spectrometry. Results: The product analysis by the three different complementary methods highlighted the presence of Carbofuran, an insecticide belonging to the carbamate family, an inhibitor of cholinesterase activity. Conclusion: The product identification allowed a fraud denunciation and drew the public authorities' attention to the illegal and unofficial marketing of these pesticides that were diverted from their initial use and that are prohibited for import in Algeria. Moreover, this identification process enabled the clinician to manage this poisoning.

Key words: Poisoning, cholinesterase symptomatology, active ingredient, chromatographic methods, identification

Reçu le 16 mars 2011, accepté après modifications le 1 juillet 2011

Publication en ligne le 17 août 2011 


\section{Introduction}

Les intoxications aux rodenticides domestiques sont en nette recrudescence ces dernières années dans la région algéroise. Ces intoxications concernent aussi bien l'adulte que l'enfant, avec un tableau clinique qui ne correspond nullement aux classes chimiques habituelles des raticides.

Chez l'adulte, ces dernières rentrent dans le cadre d'une absorption massive à but suicidaire. Chez l'enfant, il s'agit surtout des intoxications accidentelles.

Les rodenticides sont des substances actives appartenant à diverses classes chimiques. Les spécialités à activité anticoagulante sont les plus largement répandues et représentent $90 \%$ du marché. Notre étude porte sur un produit commercialisé d'une façon illicite sous le nom de « Rat Killer» depuis l'année 1990, de composition inconnue, qui a été à l'origine de 1136 cas d'intoxication d'adultes (56\%) et d'enfants (44\%) recensés au centre antipoison du centre hospitalo-universitaire de Bab El Oued d'Alger.

Le produit se présente sous forme de granulés de couleur noire, conditionnés en sachets transparents d'un poids de $2 \mathrm{mg}$ chacun. L'étiquetage diffère d'un échantillon à l'autre : certains échantillons mentionnent l'administration d'atropine en cas d'intoxication; pour d'autres, il est prescrit la prise de vitamine K. Nos patients se sont présentés avec une symptomatologie typique des insecticides anticholinestérasiques, à savoir des signes muscariniques et nicotiniques. Devant cette ambiguiité, notre objectif a été d'identifier la matière active de ce produit par des techniques sensibles et performantes afin d'élucider cette énigme.

\section{Matériels et méthodes}

\subsection{Réactifs}

L'acétone et l'acétate d'éthyle sont de grade analytique. L'acétone est fourni par Panreac et l'acétate d'éthyle par Merck. Le malathion, utilisé comme étalon interne, provient de Symrise GmbH et Co.KG. Le Fast blue a été fourni par BDH Chemicals LTD. Acétonitrile et méthanol sont de grade HPLC et d'origine respective Panreac et Fluka. L'acide formique a été fourni par Riedel-de Haën et le Formiate d'ammonium par Sigma Aldrich. L'eau est de qualité millipore, utilisée pour la préparation de la phase mobile aqueuse et des solutions aqueuses.

\section{2 Échantillons}

Un échantillonnage a été effectué sur 24 boîtes de Rat Killer en provenance de trois distributeurs : le premier situé à Alger (Dar El Beida), le deuxième dans la banlieue d'Alger (Baraki) et le troisième au sud algérien (Ghardaïa).

- Préparation de trois solutions stock à $1000 \mu \mathrm{g} / \mathrm{mL}$ par dissolution de $5 \mathrm{mg}$ de produit dans $5 \mathrm{~mL}$ de méthanol :

- solution (A) à $1000 \mu \mathrm{g} / \mathrm{mL}$ de produit Rat killer en provenance de Dar El Beida;
- solution (B) à $1000 \mu \mathrm{g} / \mathrm{mL}$ de produit Rat killer en provenance de Baraki;

- solution (C) à $1000 \mu \mathrm{g} / \mathrm{mL}$ de produit Rat killer en provenance de Ghardaïa.

- Préparation de trois solutions filles (A), (B), (C) à $100 \mu \mathrm{g} / \mathrm{mL}$.

Ces échantillons ont été analysés par des méthodes chromatographiques, à savoir chromatographie sur couche mince (CCM), afin de déterminer l'inhibition de l'activité cholinestérasique, puis par chromatographie en phase gazeuse couplée à la spectrométrie de masse (CPG/MS) permettant de fournir des informations structurales sur ces échantillons, et enfin une confirmation des résultats par chromatographie liquide couplée à la spectrométrie de masse (LC/MS/MS) 3200QTRAP.

\subsection{Conditions d'analyse}

\subsubsection{Conditions d'analyse par CCM [1]}

- Activer la plaque de CCM dans l'étuve pendant une heure à $100^{\circ} \mathrm{C}$.

- Saturer la cuve d'élution avec le mélange acétate d'éthyle/acétone $(25 \mathrm{~V} / 3 \mathrm{~V})$.

- Déposer à environ $3 \mathrm{~cm}$ du bord inférieur de la plaque $100 \mu \mathrm{L}$ des trois échantillons (A), (B), (C) préparés et $100 \mu \mathrm{L}$ de malathion utilisé comme étalon interne.

La révélation s'est faite par voie enzymatique par broyage du foie d'agneau riche en enzymes cholinestérases à $-4{ }^{\circ} \mathrm{C}$ dans de la glace. L'extrait d'enzymes est filtré.

- Pulvériser $(20 \mathrm{~mL})$ de cet extrait sur la plaque et laisser incuber dans l'étuve à $37^{\circ} \mathrm{C}$ pendant 30 min.

- Pulvériser la plaque avec le mélange préparé extemporanément : acètate de naphtyl/éthanol $(20 \mathrm{mg} / 8 \mathrm{~mL})$ et le Fast Blue/eau (50 mg/32 mL).

- Placer la plaque à l'étuve à $37^{\circ} \mathrm{C}$ pendant $10 \mathrm{~min}$.

\subsubsection{Conditions d'analyse par CPG/MS}

- Détecteur quadripôle avec source à impact électronique (EI) ;

- colonne capillaire Elit $5 \mathrm{~ms}(0,25 \mu \mathrm{m} \times 0,25 \mu \mathrm{m} \times 30 \mathrm{~m})$;

- débit de l'hélium : $1 \mathrm{~mL} / \mathrm{min}$;

- température de l'injecteur : $280{ }^{\circ} \mathrm{C}$ en mode Splitless ;

- température de la ligne de transfert : $280^{\circ} \mathrm{C}$;

- température de la source d'ionisation : $200{ }^{\circ} \mathrm{C}$;

- énergie d'ionisation à impact électronique : $70 \mathrm{eV}$;

- la température initiale de la colonne est maintenue à $100^{\circ} \mathrm{C}$ pendant $1 \mathrm{~min}$, puis programmée d'augmenter en deux paliers : un premier palier, à raison de $25^{\circ} \mathrm{C} / \mathrm{min}$ jusqu'à $150{ }^{\circ} \mathrm{C}$, température maintenue pendant $10 \mathrm{~min}$; un deuxième palier, à raison de $8^{\circ} \mathrm{C} / \mathrm{min}$ jusqu'à $300^{\circ} \mathrm{C}$, température stabilisée pendant $10 \mathrm{~min}$;

- volume d'injection : $1 \mu \mathrm{L}$

- mélange de $5 \mathrm{mg}$ de l'échantillon (A), puis l'échantillon (B) et l'échantillon (C) sont préparés dans $5 \mathrm{~mL}$ de méthanol et injectés ;

- mode : SIM (Single Ion Monitoring). 


\subsubsection{Conditions d'analyse par chromatographie en phase liquide couplée à la spectrométrie de masse en tandem (LC/MS/MS)}

\section{Chromatographie liquide (LC)}

- Chromatographe de marque Perkin Elmer;

- colonne phenomenex Luna $5 \mu$;

$-\mathrm{C} 18(50 \mathrm{~mm} \times 2 \mathrm{~mm})$ à $25^{\circ} \mathrm{C}$;

- phase mobile constituée de :

- phase A (eau+formiate d'ammonium $2 \mathrm{mM}$ + acide formique $0,2 \%)$;

- phase B (acétonitrile + formiate d'ammonium $2 \mathrm{mM}+$ acide formique $0,2 \%$ ) en mode gradient (tableau 1 );

Tableau I. Programmation de la phase mobile.

\begin{tabular}{l|ccc}
\hline $\begin{array}{l}\text { Temps } \\
(\min )\end{array}$ & $\begin{array}{c}\text { Phase A } \\
\%\end{array}$ & $\begin{array}{c}\text { Phase B } \\
\%\end{array}$ & $\begin{array}{c}\text { Débit } \\
\mu \mathrm{L} / \mathrm{min}\end{array}$ \\
\hline 5 & 80 & 20 & 250 \\
\hline 8 & 10 & 90 & 250 \\
\hline 14 & 10 & 10 & 250 \\
\hline 15 & 80 & 20 & 250 \\
\hline
\end{tabular}

- volume injecté : $10 \mu \mathrm{L}$.

\section{Spectrométrie de masse 3200QTrap}

- Mode d'ionisation positif;

- les paramètres de la source ont été optimisés selon le tableau 2;

Tableau II. Optimisation des paramètres de la source.

\begin{tabular}{l|l}
\hline Curtain gaz (CUR) & $15 \mathrm{psi}$ \\
\hline Collision gaz (CAD) & High \\
\hline Source température & $500^{\circ} \mathrm{C}$ \\
\hline Nebulizer gaz (GS1) & $40 \mathrm{psi}$ \\
\hline Auxiliary gaz (GS2) & $60 \mathrm{psi}$ \\
\hline Interface heater (Ihe) & On \\
\hline
\end{tabular}

- mode : Multiple réaction monitoring (MRM).

\section{Résultats}

\subsection{Chromatographie sur couche mince}

La chromatographie sur couche mince met en évidence des RF identiques aussi bien pour les échantillons $\mathrm{A}, \mathrm{B}, \mathrm{C}$ que pour l'étalon interne. La présence de quatre taches blanchâtres démontre une inhibition de l'activité cholinestérasique (figure 1).

Il s'agit d'un pesticide appartenant soit au groupe des organophosphorés soit au groupe des carbamates.

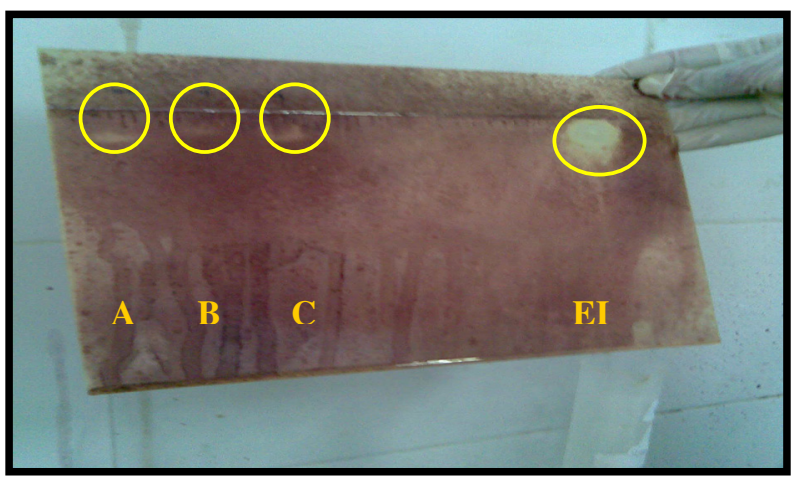

Fig. 1. Inhibition de l'activité cholinéstérasique par CCM : A : Rat Killer Dar el Beida ; B : Rat Killer Baraki ; C : Rat Killer Ghardaia ; EI : Malathion.

\subsection{Chromatographie en phase gazeuse couplée à la spectrométrie de masse}

Les chromatogrammes obtenus par $\mathrm{CPG} / \mathrm{MS}$ révèlent la présence d'un pic correspondant à une molécule éluée à $8,36 \pm 0,01 \mathrm{~min}$ (figure 2).

Le spectre de masse de cette molécule identifie la présence de deux fragments (164) et (149) retrouvés dans les trois échantillons, relatifs à la présence du carbofuran par comparaison avec les spectres de référence de la librairie incorporée (figure 3).

\subsection{Chromatographie en phase liquide couplée à la spectrométrie de masse en tandem}

L'analyse par LC/MS/MS relève deux transitions MRM parents/fils $(222,1 / 165,2)$ et $(222,1$ et 123$)$. Ces deux transitions ont été comparées aux 94 transitions de la librairie carbamates, confirmant la présence du carbofuran (figure 4).

\section{Discussion}

Ces dix dernières années (1998-2008), l'intoxication aiguë au Rat Killer a représenté $51 \%$ des intoxications aux raticides selon les données du centre Antipoison de Bab El Oued d'Alger; ceci en raison de sa large commercialisation et de son coût modique, évalué à 10 dinars la boîte $(0,1$ centime d'euro). Les premières intoxications au Rat Killer ont été décelées une année après sa commercialisation, en 1998.

Depuis, une ascension fulgurante a été observée (figure 5). Les intoxications d'origine suicidaire sont retrouvées dans $56 \%$.

Les tranches d'âge, comprises entre 21 et 40 ans, sont les plus exposées. Les intoxications accidentelles chez les enfants sont observées dans $44 \%$ des cas et la tranche d'âge 04 ans est la plus affectée. Les manifestations cliniques étaient asymptomatiques dans $43 \%$ des cas, le syndrome cholinergique retrouvé dans $57 \%$ des cas.

Des résultats analogues ont été relevés au service de réanimation du CHU Annaba, situé dans la région Est d'Algérie, 

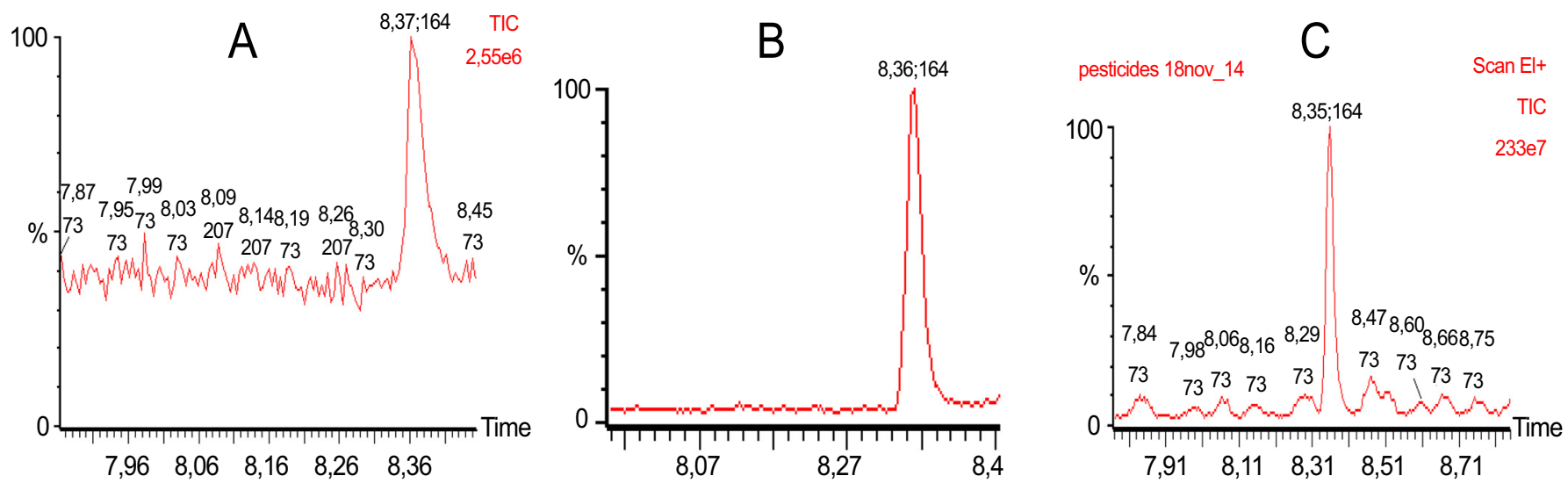

Fig. 2. Chromatogrammes CPG/SM : A : Rat Killer Dar el Beida, B : Rat Killer Baraki ; C : Rat Killer Ghardaia, 1 mg/mL.
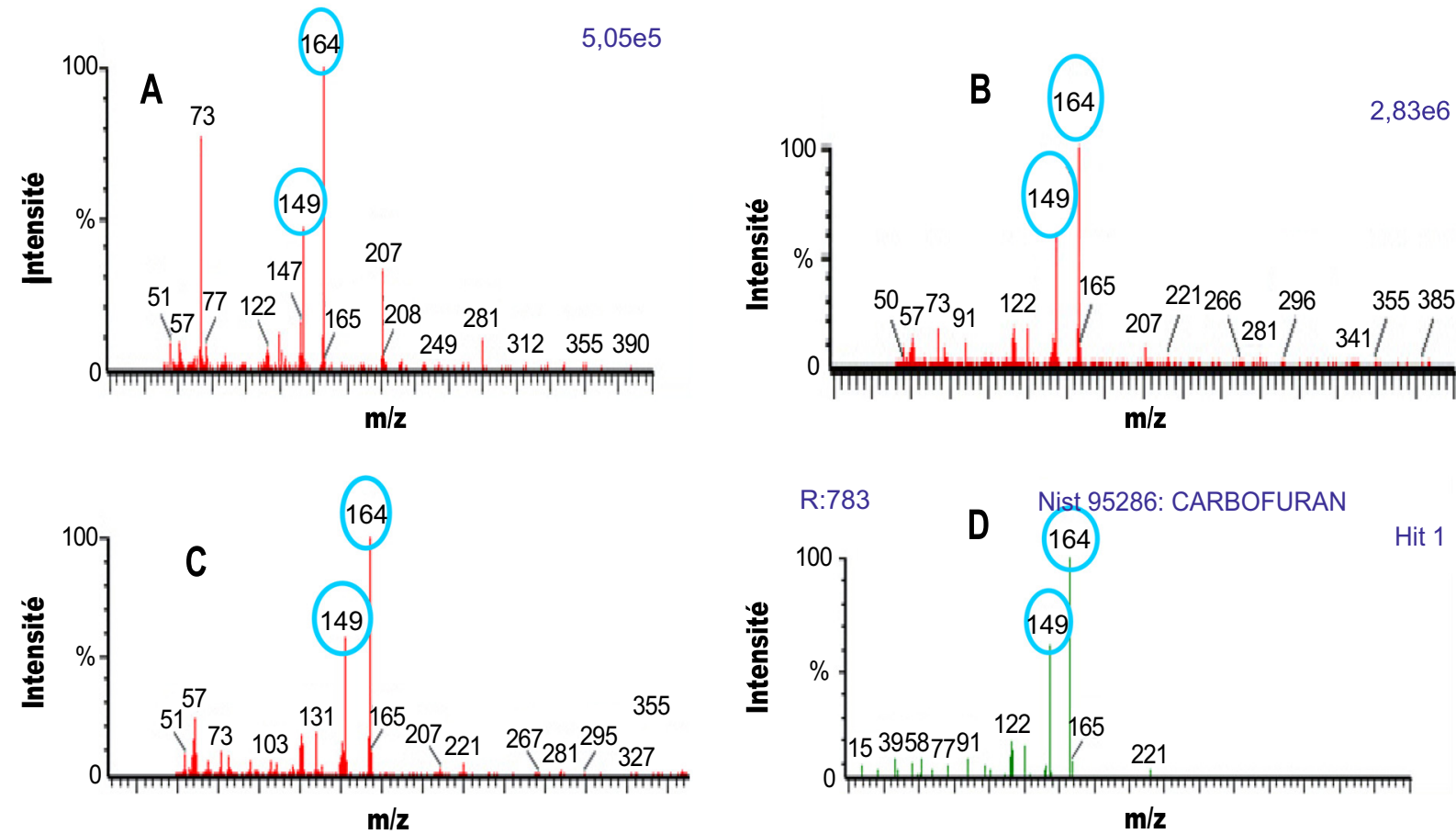

Fig. 3. Spectres de masse CPG / SM : A :Rat Killer Dar el Beida; B : Rat Killer Baraki : C : Rat Killer Ghardaia ; D : spectre de référence de la Librarie Nist incorporée. Sont représentés des fragments 164/149 correspondant au carbofuran $1 \mathrm{mg} / \mathrm{mL}$.

où une fréquence beaucoup plus élevée a été trouvée (85\%) durant la période 2004-2007 (données non publiées).

Par ailleurs, 152 cas d'intoxication ont pu être colligés au centre hospitalier d'Oran, situé dans la région Ouest d'Algérie durant les années 2005-2007. La symptomatologie clinique des rodenticides diffère d'une famille à une autre. Pour les rodenticides anticoagulants ou anti-vitamine K, les symptômes d'hémorragies apparaissent après quelques jours pour une dose élevée, après quelques semaines pour des prises répétées [2]. Ces signes cliniques n'ont pas été observés chez nos patients. Des signes muscariniques (bradycardie, myosis, hypersécrétion bronchique, vomissement, diarrhées) et des signes nicotiniques (asthénie, fasciculations musculaires) ont été relevés au centre antipoison du CHU Bab El Oued.
Ces données ont aussi été rapportées par l'étude réalisée au service de réanimation du CHU Annaba, où des fréquences de $22,5 \%$ pour le syndrome muscarinique et $11,28 \%$ pour le syndrome nicotinique ont été retrouvés.

Des résultats analogues ont aussi été relevés par les travaux effectués au centre hospitalier d'Oran (données non publiées) : une bradycardie (19\% des cas), un myosis (70\%), une hypersécrétion bronchique, des vomissements et diarrhées (89\%), une myasthénie (16\%) et des fasciculations musculaires (4\%). Ce tableau très évocateur est lié à l'inondation cholinergique de l'organisme due à l'inhibition des cholinestérases [3-5].

L'analyse du produit par les techniques chromatographiques confirme la présence du carbofuran, qui est un carbamate, un inhibiteur de l'activité cholinéstérasique justifiant 

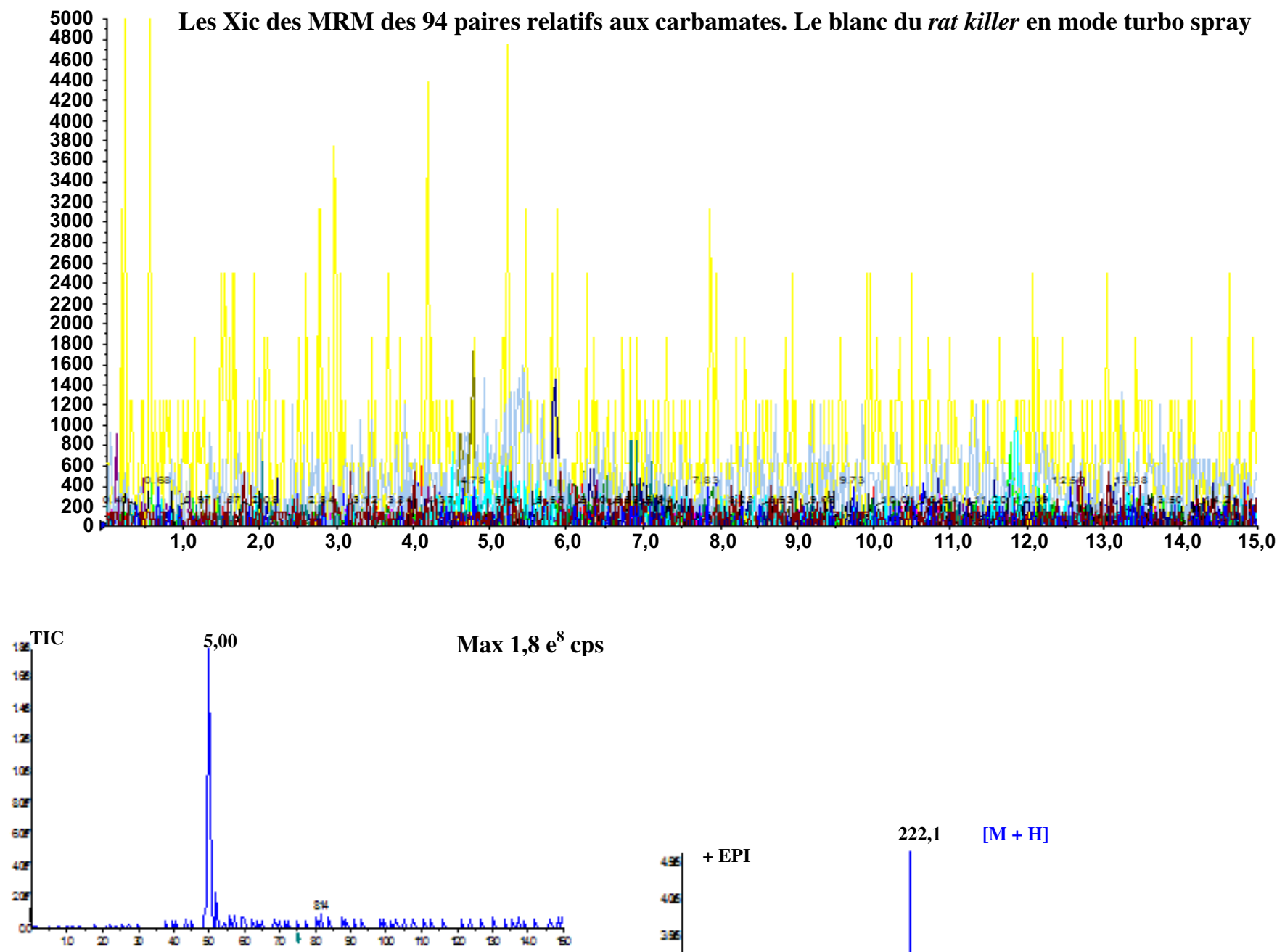

temps
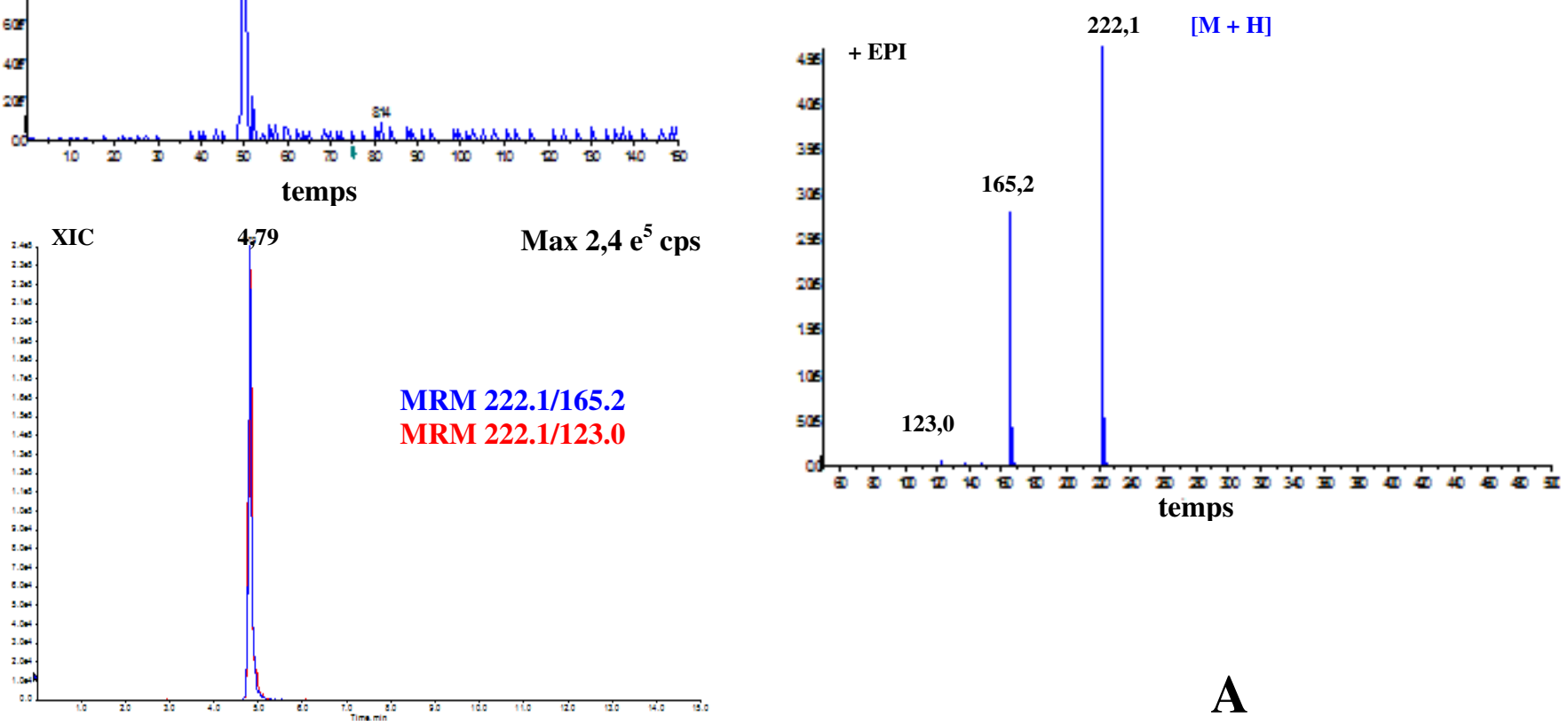

temps

Fig. 4. Blanc, chromatogramme total, chromatogramme extrait du carbofuran (MRM 222,1/165,2 et 222,1/123,0) et spectre de masse des trois échantillons : A : Rat killer Dar el Beida; B : Rat killer Baraki ; C : Rat killer Ghardaia. 

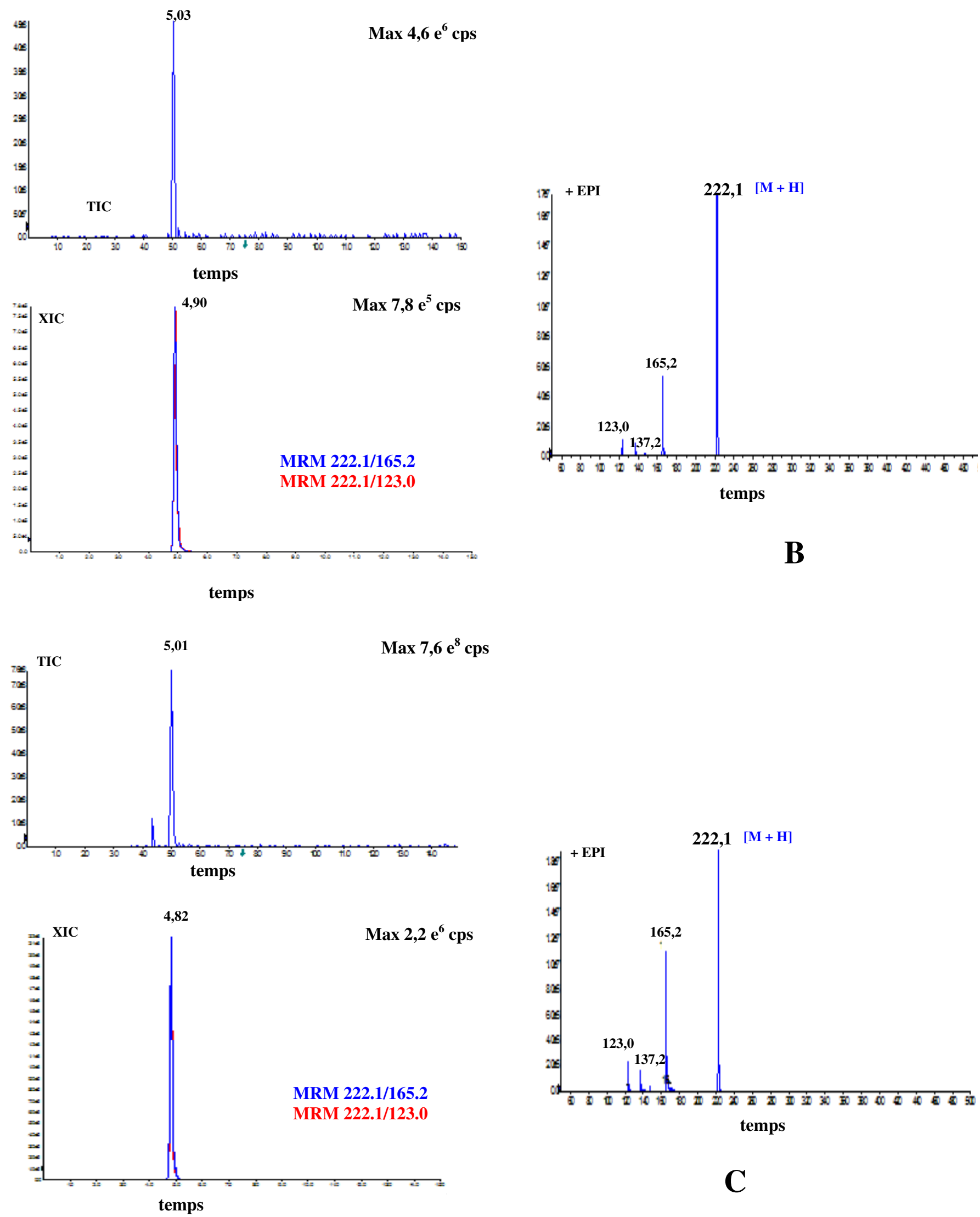

Fig. 4. Suite. 




Fig. 5. Répartition des intoxications au Rat Killer sur une période de dix ans (1998-2008).

Tableau III. Traitement en fonction de la gravité de l'intoxication.

\begin{tabular}{l|ll}
\hline $\begin{array}{l}\text { Signes } \\
\text { cliniques }\end{array}$ & $\begin{array}{l}\text { Taux de } \\
\text { cholinestérases }\end{array}$ & Traitement \\
\hline Présents & Très effondré & $\begin{array}{l}\text { - Lavage gastrique } \\
\text { - Administration d'atropine } \\
\text { - Contrôle cholinestérases }\end{array}$ \\
\hline Présents & Dans les normes & $\begin{array}{l}\text { - Lavage gastrique } \\
\text { - Administration d'atropine }\end{array}$ \\
\hline Absents & Bas & Contrôle des cholinestérases \\
\hline Absents & Dans les normes & Abstention \\
\hline
\end{tabular}

bien la symptomatologie clinique citée ci-dessus [6]. Il importe de rappeler que le carbofuran est un pesticide qui a été détourné de son usage insecticide pour être utilisé comme rodenticide.

Sur le plan de la réglementation des produits phytosanitaires, le carbofuran est une matière active non homologuée en Algérie, selon la base de données du ministère de l'agriculture et de la pêche [7].

Les signes cliniques d'inhibition cholinestérasiques apparaissent pour une dose orale unique de $0,1 \mathrm{mg} / \mathrm{kg}$ de poids corporel [8].

Suite à l'identification du produit, le centre antipoison d'Alger a instauré une conduite à tenir devant d'éventuelles intoxications et élaboré un traitement adéquat en fonction de la gravité de l'intoxication.

Le traitement symptomatique est le suivant :

- lavage gastrique :

- intubation/ventilation si nécessaire, aspiration des sécrétions bronchiques, correction d'une acidose et des convulsions.

Le traitement spécifique consiste en une administration de l'atropine corrigeant les signes muscariniques, mais elle est sans effet sur les signes nicotiniques et sur les cholinestérases. L'administration initiale d'une dose de 1 à $2 \mathrm{mg}$ chez l'adulte, soit $0,01-0,05 \mathrm{mg} / \mathrm{kg}$ chez l'enfant par voie IV, répétée toutes les 10 à 30 minutes jusqu'à l'obtention de signes d'atropinisation, tout en évitant la possibilité d'une intoxication atropinique.

\section{Conclusion}

L'intoxication par le Rat killer est relativement grave, bien qu'aucun décès n'ait été rapporté. Par ailleurs, l'importance des techniques chromatographiques, notamment la LC/MS/MS en tandem, a permis d'une part d'identifier la matière active en vue d'orienter le clinicien vers l'élaboration d'un traitement adéquat et d'autre part d'interpeller les pouvoirs publics quant à la commercialisation frauduleuse et informelle de ces pesticides interdits à l'importation, qui ont été détournés de leur usage initial.

Remerciements. Nous remercions madame le Professeur Rachida Merad, qui a bien voulu relire et corriger cet article.

Conflits d'intérêts. Les auteurs déclarent ne pas avoir de conflits d'intérêts.

\section{Références}

1. Erdmann et al. Thin layer chromatography in standardized systems. 1990, Sunshine 1975, DFG /TIAFT 1992: 12-20.

2. Gamelin L, Harry P. Rodenticides. Centre Anti Poison Interrégional. Elsevier 2005; 2(3): 89

3. Pinakini KS, Mohan Kumar T.S. Serial cholinesterase estimation in carbamate poisoning. J clin Forensic Med. 2006; 13: 274276.

4. Testud F. Les carbamates anti cholinestérasiques. In : Testud F, Garnier R, Delemotte B (coordinateur). Toxicologie humaine des produits phytosanitaires. Paris: ESKA et LACASSAGNE. 2001: 91-101.

5. Vale JA. Les carbamates insecticides. Intoxications par les insecticides organophosphorés et par les carbamates In : Jaeger A, Vale JA (coordinateur). Intoxications aiguës. Paris: Elsevier. 1999: 431-447.

6. Ferslew KE, Hagardorn AN, Mc Cormick WF. Poisoning from oral ingestion of carbofuran, a cholinesterase-inhibiting carbamates insecticide, and its effects on cholinesterase activity in various biological fluids. J Forensic Sci. 1992; 37: 337-344.

7. Direction de la protection des végétaux et des contrôles techniques. Index des produits phytosanitaires à usage agricole. Édition 2007: 34-97.

8. Afssa - Agritox : Base de données sur les substances actives phyto pharmaceutique. Document consulté sur le site : http://dive-afssa.fr/agritox/indexphp, le 13 juin 2011. 